\title{
ANÁLISIS FENOMENOLÓGICO DE LA DEDUCCIÓN TRASCENDENTAL DE KANT \\ (Fragmento)
}

\author{
Javier SAN MARTÍN
}

UNED

RESUMEN: El trabajo que presento es un fragmento de un análisis fenomenológico de la "Deducción trascendental de las categorias" de la Critica de la razón pura de Kant. Esas páginas del libro más importante de Kant son la clave de todo el edificio critico. Su dificultad aconseja leerlas con detenimiento. En este fragmento, que se limita a la primera sección de la deducción, los $5 S 13$ y 14 en la segunda edición de la Crítica, se trata de alcanzar, mediante un método fenomenológico, la situación de experiencia que cada expresión quiere reflejar. Con ese método se descubre la importancia de pensar la noción de "concepto empirico", que siendo necesaria para comprender la de "concepto puro" suele pasar desapercibida. A lo largo de esta lectura como "ejercicio de análisis fenomenológico" podremos introducirnos en la forma de argumentación de Kant, accediendo a la comprensión de algunos de sus puntos más dificiles, poniéndose de relieve la dificultad de la existencia de conceptos como pensamientos de objetos al margen de la experiencia, así como el sentido básico y el alcance de la deducción trascendental.

The paper is a fragment of a phenomenological analysis of the "transcendental Deduction" of Kant's Critic of pure Reason. Those pages of Kant's most important book are the key for the whole critical construction. Their difficulty suggests a carefully reading of them. The fragment, which is limited only to the first section of the Deduction, the SS 13 and 14 in the second edition of the Critic, aims the situation of experience of every expression by means of a phenomenological method. By this method the importance of the "empirical concepts" is unveiled; those concepts are necessary for thinking the "pure concepts" but remain usually without taking into account. Along this reading as "exercise of phenomenological analysis" we can introduce ourselves in Kant's form of arguing, and so reach an understanding of some difficult points of the Critic, bringing into relief the difficulty of concepts as thoughts of objects out of the experience, as well as the basic meaning and importance of the transcendental Deduction 


\section{A modo de presentación}

El siguiente texto ofrece las dos primeras secciones de un amplio capítulo para un libro que se titularía "Problemas y ejercicios de fenomenología». El libro tiene previsto publicar textos de fenomenología de hace unos treinta años y que sirvieron, en su momento, de "ejercicios", en sentido escolar, o para dar vueltas a algunos problemas con los que el autor se encontraba en el estudio de la fenomenología. El presente responde más al de «ejercicios» de fenomenología. Su origen está en la lectura fenomenológica que hizo de la "Deducción trascendental de las categorías", de la Crítica de la razón pura de Kant, en la asignatura de Teoría del Conocimiento, que el autor impartió en la Facultad de Filosofía de Santiago de Compostela, según sus datos, el curso 1977-78. En él no se llegó al final del comentario, que quedó escrito en tres cuadernos tamaño cuartilla. Uno de ellos, sin embargo, lo extravió el autor en el calle, y de él sólo pudo recuperar una parte, faltándole las primeras siete hojas. El fragmento que aquí se publica es el comentario a la parte del texto que en segunda edición de la crítica queda enmarcada como 13 y 14. Su objetivo, además de ser un "ejercicio" de fenomenología, puede servir para incentivar a los lectores a acercarse a Kant, autor que, leído con el debido detenimiento, es una fuente inagotable para la formación filosófica. La aplicación de una lectura fenomenológica, buscando siempre, como diŕa Fernando Montero Moliner, el "fenómeno original" que está detrás de las expresiones, puede ayudar a penetrar en la exposición kantiana.

\section{Introducción}

La historia de la filosoffa intenta sistematizar la marcha de los diversos sistemas filosóficos; para ello hay que tratar de comprender el verdadero sentido de un autor. Mas en la medida en que ese autor aporte algo que nos interpela filosbficamente de modo continuo, la historia de la filosofia es o ha de ser filosofia. No se trata entonces, en ella, de una mera repetición de lo que dijeron el uno o el otro, sino de captar la verdadera incidencia de sus planteamientos en la filosofla como conjunto tradicional de pensamientos, desglosándolos de todo lo accidental y contingente. De ahí que necesitemos ir a la profundidad del pensar o, como diría Heidegger, a la cosa del pensamiento, a las verdaderas intuiciones filosóficas.

Mi objetivo es contribuir a estudiar la "Deducción trascendental" de la Critica de la razón pura de Kant, verificando sus afirmaciones y desarrollo. La dificultad de 
esas páginas aconseja aplicar un método eficaz; en estas páginas propongo utilizar el método fenomenológico. La práctica misma de la interpretación nos familiarizará con algunas de las características del mismo, por lo que no hace falta exponer en qué consiste. Nuestro objetivo es ver, como lo requiere el método fenomenológico, a qué corresponde cada expresión, dando por supuesto que cada una ha de serlo de un fenómeno o que éste ha de estar intencionalmente implicado por el mencionado en la expresión, es decir, ha de venir exigido intencionalmente por el fenómeno directamente mencionado, como la apertura de una puerta para salir de la habitación implica la continuidad del suelo debajo de nuestros pies. Si encontramos afirmaciones para las que no hallamos un fenómeno, pudieran ser afirmaciones metafísicas o especulativas, que pueden responder a una opción en la comprensión del mundo, pero en ningún caso fenomenológicamente justificables.

Creemos en la eficacia del método fenomenológico. La fenomenología de Husserl pretende la descripción de los fenómenos que constituyen nuestra vida consciente; sus afirmaciones son descripciones de fenómenos. Kant puede ser abordado con un bagaje fenomenológico de modo fructifero. No intentaremos una comparación externa de Kant y Husserl, una comparación de sus motivaciones, metas, diversidad de métodos etc. Sólo intentaremos analizar fenomenológicamente, en este caso desde Husserl, una parte nuclear del pensamiento crítico de Kant como base de una teoría del conocimiento. Una parte muy importante de ese pensamiento viene expresada en la "deducción trascendental de las categorías". Vamos a leer a Kant dejándonos llevar por él, pero a la vez mirando cada afirmación con detenimiento, para descubrir todos los posibles fenómenos a que él mismo se podía referir. Al principio quizás sea penoso o difícil ver si a determinadas afirmaciones corresponde un fenómeno; pero la práctica de clarificación fenomenológica de un texto terminará por introducir en el lector el hábito de buscar para el lenguaje la base fenomenológica de las afirmaciones. En ese sentido el texto es un «ejercicio de fenomenología». En todo caso valdrán estas páginas como un experimento con un texto especialmente difícil, con lo que me adhiero al interés que Eugen Fink mostró en estos textos. Tengo la esperanza de que este ejercicio interese y sirva, primero, para una introducción a la teoría del conocimiento; segundo, para introducir a Kant; y, en tercer lugar, como adquisición de una técnica interpretativa, que, a la larga, pudiera reportar beneficios.

Hay que señalar por dónde vamos a empezar o qué parte de la Crítica de la razón pura hemos elegido para este experimento o ejercicio de interpretación fenomenológica. Como se puede ver en el esquema adjunto, sólo vamos a tratar 
o someter a este experimento de comentario el capítulo segundo del libro primero de la "Analítica trascendental". Aunque se trata de un capítulo reducido, sin embargo constituye en gran medida el núcleo de todo el pensamiento kantiano, el más comprometido para él y además de no fácil lectura.

\section{CRITICA DE LA RAZÓN PURA}

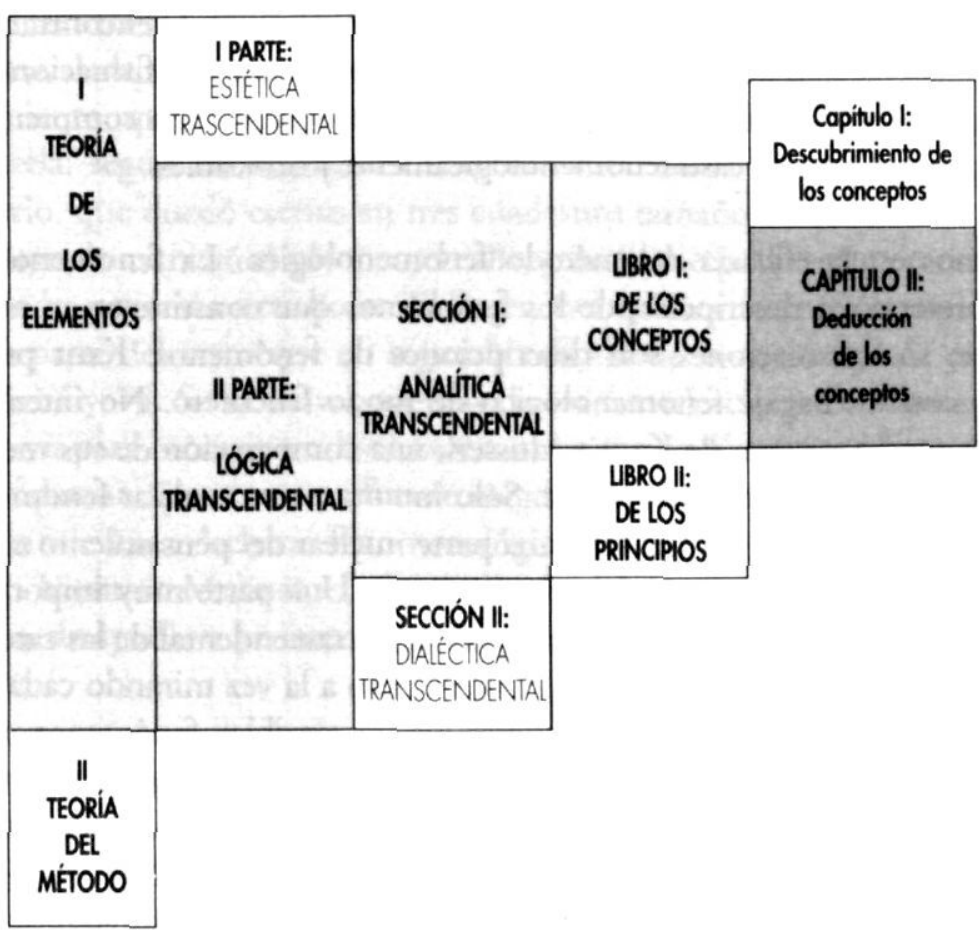

Si se entiende bien esta parte, el resto de la filosofía de Kant puede resultar relativamente más asequible. El capítulo está dividido en dos secciones, la primera abarca los $\$ \$ 13$ y 14 , la segunda los $\$ \$ 15$ a 27 . Hay que advertir que la distribución en parágrafos proviene del cambio de la segunda edición, pues no se da en la primera. En este texto se comentarán solamente los $\$ \$ 13$ y 14 . 


\section{El $\S 13$ : sobre los principios de una deducción trascendental en general}

\subsection{Qué es la deducción}

Kant empieza definiendo qué es una deducción en términos jurídicos: la exigencia de pruebas sobre un derecho. En términos jurídicos significa la presentación de pruebas sobre el derecho que uno tiene a algo. Si afirmo que tengo derecho al trabajo, aunque de hecho no trabaje porque no hay puesto para mí, la deducción es la aportación de pruebas sobre ese derecho, porque de esas pruebas "se deduce" el derecho'. En las deducciones jurídicas las pruebas que se aportan son las leyes que asignan ese derecho. Pero utilizamos en otros muchos casos conceptos. ¿Cuáles son las pruebas que se pueden aducir para justificar expresiones del tipo «los hombres tienden a la felicidad», o cualquier otra expresión en la cual haya algún concepto? Kant nos dice que utilizamos «eine Menge empirischer Begriffe», una cantidad de conceptos empíricos. Para el uso de estos conceptos empíricos (punto clave de la teoría de Kant) tenemos a mano la experiencia; las pruebas que puedo aportar para la legitimidad del concepto de felicidad en la frase anteriormente indicada son las series de experiencias personales y sociales en las cuales vemos, en efecto, que las diversas acciones de los hombres y de los grupos se realizan para alcanzar algún grado de satisfacción, a la que solemos llamar felicidad. Otro problema será ver en qué tipos de satisfacción ponen los diversos pueblos la felicidad; mientras unos creerán que la felicidad radica en la satisfacción de necesidades corporales, alimento y sexo principalmente, para otros radicará en la satisfacción intelectual, mientras que otros considerarán que el dominio del propio cuerpo y de los intereses generales de la vida son el contenido fundamental de la felicidad.

Kant habla por tanto de "conceptos empíricos". Uno de nuestros principales objetivos será tomar cabal conciencia de esta noción. Pues bien, en el caso de los "conceptos empíricos" la "realidad objetiva del concepto es esa experiencia" aportada por la deducción en aras de su justificación. Es importante ver estas

1 Sería interesante anotar aquí lo difícil que resulta justificar nuestros derechos en otros términos que las concesiones; tengo derecho al trabajo porque la sociedad me lo da; una pregunta de gran interés para la antropología es si se puede dar una razón estrictamente antropológica, es decir, basada en la naturaleza del ser humano más que en las características de la sociedad. 
distinciones introducidas por Kant; en efecto, Kant habla de experiencia [Erfahrung], realidad objetiva [objektive Realität] y conceptos [Begriffe]. La conexión que de momento vemos entre estas tres palabras se puede formular con la siguiente proposición: la realidad objetiva de un concepto no puede ser otra cosa que el hecho de que ese concepto corresponda a lo dado en la experiencia. El desarrollo de este comentario irá clarificando toda problemática que se encierra en esa formulación.

\subsection{El concepto empírico}

Antes de seguir con nuestro comentario conviene clarificar un punto decisivo en la teoría del conocimiento de Kant, que probablemente hoy ya esté solucionado en la filosofía. Me refiero a la relación entre el concepto y la palabra, aspecto que, propiamente hablando, Kant no toma en consideración. ¿Qué se designa con la palabra, el concepto, o las experiencias que constituyen lo que hemos llamado realidad objetiva del concepto? Tradicionalmente, y creemos que Kant aceptó esa tradición, la palabra designa el concepto y éste tiene una realidad objetiva si se dan las experiencias sensibles a que se refiere. Es importante preguntarse, para iniciar ya la práctica de nuestra interpretación o lectura fenomenológica, qué experiencia o fenómeno es descrito en esta concepción del lenguaje. Es muy importante aclararlo ya desde ahora, pues ello va a tener muchisima importancia en Kant, pues al aceptar Kant esta separación entre la realidad y la palabra, entre las cuales se introduce el concepto, tendrá vía libre para aceptar un pensamiento puro libre de la experiencia, en definitiva, para aceptar un modelo metafísico del ser humano. Curiosamente iremos viendo que la deducción trascendental supera ese modelo metafísico, o que lo lleva hacia otros derroteros, hacia derroteros que podríamos llamar fenomenológico-materialistas, aunque el propio Kant lo rectificara en la segunda edición de la Crítica.

Supongamos que el concepto tiene realidad objetiva y que con la palabra se designa el concepto. Estamos hablando de los "conceptos empíricos", es decir, de aquellos cuyas pruebas de validez se pueden encontrar citando o aportando la experiencia. En este caso ¿qué es lo que podemos decir del concepto empírico? ¿qué puede ser el concepto empírico? ¿en qué se diferencia de la experiencia concreta y actual? ¡existe este concepto empírico distinto de las palabras que lo designan? ¿o designan las palabras la experiencia y no los conceptos? El término "concepto empírico" es una de las grandes aportaciones de Kant, plenamente justificable desde una perspectiva fenomenológica. Con esto queremos decir que 
hay efectivamente una situación fenomenológica que queda descrita por esa palabra «concepto empíricom. Entendemos por concepto empírico la serie de experiencias sintetizadas y normalmente simplificadas por la repetición de la experiencia de lo común y retenidas en mi conciencia, en el sentido fenomenológico de la palabra 'retención'. El concepto es un conjunto de experiencias, temporalizado en la forma de pasado, perteneciente a mi conciencia presente. A este conjunto de experiencias implicadas (concepto) y al cual corresponde un objeto en la experiencia, teniendo así "realidad objetiva", designamos con una palabra, que por ello designa tanto al concepto como a la experiencia de un objeto. Sin embargo, Kant no parte de aquí sino de una idea intelectualizada de concepto, pero que será superada indefectiblemente en la «deducción». Conviene reflexionar sobre este punto, pues es uno de los más decisivos en la teoría del conocimiento: el lugar del concepto, de la experiencia y del objeto. En este comentario intentaremos aclarar estos términos.

\subsection{Deducción de los conceptos puros}

Respecto a la deducción de los conceptos empíricos, de cuya realidad objetiva podemos tenemos pruebas en la experiencia, no habría ningún problema. Se trata de aducir o aportar esa experiencia. Pero tenemos, según Kant, otros conceptos totalmente independientes de la experiencia, y aquí es donde se plantea el verdadero problema de la Crítica. Esos conceptos son usados a priori, es decir, los mencionamos de forma independiente de la experiencia, con lo cual la deducción o prueba de su realidad objetiva ya no puede hacerse presentando o aportando una experiencia. La prueba del derecho a utilizar esos conceptos no puede ser la aportación de la experiencia. Nada ayuda aportar o acumular experiencias para justificarlos, como en el Derecho nada justifica una situación de hecho, si ese hecho no está sancionado por normas. Por muchas realidades de hecho que presente, nunca justificarán algo de derecho hasta que no sea socialmente convertido en norma. Nos tenemos que detener en lo descrito en este párrafo segundo del $\$ 13$ para ver el posible sentido fenomenológico o especulativo del hecho de constar el uso de conceptos puros.

Hay que partir de la definición de concepto. Concepto es el pensamiento de un objeto, por eso los conceptos se refieren necesariamente a los objetos. Creo que es importante aceptar esta definición kantiana, por lo menos para trabajar con ella. Concepto es el pensamiento de un objeto. El pensamiento es conciencia (para Kant el pensamiento es algo espiritual); por eso en la definición de 
Kant podemos decir que el concepto es la conciencia espiritual de un objeto. Ya hemos visto (y repito que insistiremos mucho en ello) lo que son los conceptos empíricos: una serie de experiencias sintetizadas y simplificadas por la repetición de lo común; esto efectivamente equivale al pensamiento de un objeto, pero aquí no hay problema porque el objeto es un objeto de experiencia. Creo que sólo se puede entender correctamente la definición kantiana de concepto como pensamiento de un objeto desde una perspectiva fenomenológica. Creo que también en el desarrollo de este comentario aclararemos esta definición.

Pero aquí nos viene ahora el problema; si el concepto es el pensamiento de un objeto, ¿qué objeto pueden pensar los conceptos puros, si por definición no se refieren a objetos de la experiencia? Por definición los conceptos puros no piensan ningún objeto de la experiencia. Entonces ¿¿de dónde pueden ser esos objetos pensados por los conceptos puros? ¿Puede haber conceptos puros? En todo caso, si nos ponemos en la situación de Kant, que parte de la existencia efectiva de conceptos puros, tenemos que decir que la "deducción" de esos conceptos no puede ser empírica, porque de ellos no hay experiencia, sino trascendental. De nada me serviría para justificar estos conceptos aducir pruebas de la experiencia, puesto que nada piensan de la experiencia. En la situación fenomenológica del concepto empírico antes descrita encaja perfectamente la definición de concepto. Que el concepto piensa un objeto significa exclusivamente la presencia en la actualidad de una serie de experiencias pasadas o posibles implicadas en un dato presente o representificado. En cambio, con la afirmación del concepto puro, tendríamos un pensamiento de objetos no implicados en ningún dato presente o representificado de la experiencia, puesto que son conceptos independientes de ella. Por ejemplo, la causalidad piensa algo que no se puede dar en la experiencia puesto que en la experiencia sólo tenemos la sucesión de dos datos, pero nunca la causalidad, con el sentido conceptual que ésta tiene.

Aquí evidentemente podríamos cortar un poco el hilo de la argumentación, como lo haría Locke o un Hume y decir sencillamente que no hay tales conceptos puros, porque siempre han de designar algo de la experiencia. En el concepto de causalidad todo su contenido será precisamente esa sucesión; si la experiencia no nos da nada más que la sucesión, ése es entonces el único contenido fenomenológico de la causalidad, y si es el único contenido fenomenológico, es el único contenido justificable. Todo el resto que acoplemos a ese concepto, por ejemplo, al concepto de causalidad, o bien proviene de otras experiencias que, en todo caso, habrá que justificar en virtud de qué se le asocia a ese concepto, o 
bien son puras palabras que parecen decir algo pero que en realidad nada dicen. Pero Kant tiene un argumento decisivo a favor de esos conceptos, a saber, la certeza de la ciencia: la ciencia se basa en esos conceptos y la ciencia viene ratificada por sus éxitos prácticos, que se basan en su capacidad de predicción. De la ciencia no se puede dudar. Sin embargo es también evidente que esos conceptos no se pueden reducir a experiencias, puesto que por mucho que miremos la experiencia no vamos a encontrar otra cosa que la sucesión continua de dos fenómenos. Reducir esos conceptos a experiencia sería caer en el psicologismo.

Un concepto es el pensamiento de un objeto, es decir, un concepto debe tener siempre, si es verdadero concepto, si no es, por tanto, un mero flatus vocis, realidad objetiva. Mas ¿qué realidad objetiva puede tener un concepto puro, si por definición decimos que no se refiere a la experiencia. Por eso se le puede preguntar a Kant si estos conceptos piensan realmente un objeto. Si su objeto no está en la experiencia, ¿dónde puede estar? ¿dónde podría existir ese objeto? $\mathrm{Si}$ no piensan un objeto de la experiencia, como, por ejemplo, la justicia, que indudablemente piensa un objeto de la experiencia, o como el concepto de animal, que también piensa un objeto para la experiencia, o la felicidad, por la cual designamos una serie de experiencias muy concretas, $y$ en todo caso verificables en la experiencia, ¿qué tipo de objetos pueden pensar los conceptos puros? Según Kant los conceptos puros - aquí vienen aludidos tanto las formas del espacio y el tiempo como las categorías puras- se refieren a objetos sin tomar prestado nada de la experiencia; pero entonces ¿cuál será su contenido? Por esos conceptos pensamos algo, pero ese algo no puede ser ninguna cosa ni ninguna relación empírica de cosas. El concepto de causa presenta una necesidad que en ningún caso puede ser leída en la experiencia, o sacada de la experiencia. Lo mismo el concepto, o mejor en este caso, la forma de espacio presenta un contenido que en ningún caso puede ser reducido a la experiencia. El espacio no es alguna cosa o relación de cosas; siempre que queramos reducirlo a cosa o a relación de cosas, caeremos en contradicciones, porque tanto esa cosa como toda relación de cosas presuponen el espacio donde se dan. Es decir, el espacio es previo a toda cosa o relación de cosas. La experiencia nos da cosas, unas junto a otras; por tanto están ya en el espacio. Por eso de estos conceptos -o formassólo es posible una deducción trascendental.

De un concepto empírico cabe una deducción empírica: aducir la experiencia que ese concepto designa junto con la serie de otras experiencias implicadas en la experiencia presente. ¿Qué puedo aducir ahora para la justificación, es 
decir, para la deducción, si ya por definición estos conceptos no piensan un objeto de experiencia? Por ello concluye Kant que sólo es posible respecto a estos conceptos una deducción trascendental.

\subsection{Sentido de la «deducción trascendental»}

Si en los primeros párrafos Kant opera con la dualidad de conceptos empiricos y conceptos puros, en el caso de la deducción esa dualidad se corresponde con la contraposición entre una deducción trascendental y una deducción empírica. Ésta descubre el origen de la validez del concepto empírico, aduciendo, para ello, la experiencia. En definitiva la deducción no es más que la aportación o el descubrimiento del origen; esto es evidente en el caso del concepto empírico. Una deducción de un concepto empírico, en la medida en que me remite a la experiencia y a la serie de datos implicados en un dato presente como justificación de ese concepto, me pone en contacto con las fuentes del saber empírico. En todo caso interesa subrayar que el sentido de la deducción no es otro que buscar "la partida de nacimiento" de los conceptos. Con esto llegamos a un punto de múltiple interés. En primer lugar, vemos que la deducción no es otra cosa que la búsqueda del origen del conocimiento, en este caso, del pensamiento de objetos, o lo que es lo mismo, el origen de los conceptos. En segundo lugar, estamos ante una identificación de justificación con origen. Y en tercer lugar, en cuanto afirmamos que esta parte de la crítica es, sin duda, la más importante y básica para la teoría del conocimiento, estamos diciendo que la teoría del conocimiento tiene que buscar los origenes del conocimiento, tiene que buscar las fuentes; ésta es también la intención fundamental del método fenomenológico: ir a las fuentes, a los orígenes; en los orígenes se encuentra la justificación. Por eso Kant nos habla de que ha hecho una deducción trascendental del espacio y del tiempo mediante la cual ha perseguido estos conceptos "a sus fuentes» [zu ihren Quellen], (B, 120).

Vamos a detenernos un poco en este aspecto. Ir a las fuentes es el sentido de toda deducción; la empírica va a las fuentes de los conceptos empíricos, es decir, a las fuentes de donde surge la validez objetiva de los conceptos empíricos. Estos son un conjunto de experiencias retenidas, así en ellos se piensa un objeto de experiencia. Deducción empírica es vuelta a la experiencia en cuya fuente bebe su validez y sentido el concepto empírico. Lo peculiar de la deducción trascendental es que no puede acudir a la experiencia porque los conceptos puros no piensan un objeto de experiencia, con lo cual buscar su origen no puede ser ir a la experiencia, sino a 
fuentes independientes de la experiencia, por tanto, fuentes que son anteriores a la experiencia, fuentes que transcienden la experiencia, por eso esta deducción de los conceptos puros sólo puede ser trascendental. Por eso es una deducción a priori, a priori que hay que entender siempre en relación con la experiencia.

Ahora bien, tales conceptos son un hecho, y también hay que demostrar su validez yendo a su origen, origen que, por otro lado, no puede estar en la experiencia. Mostrar la realidad objetiva de esos conceptos será mostrar cómo pueden tener un objeto, ser objetivos, sin que su objeto esté dado en la experiencia. La dificultad aquí no está en comprender qué es lo que Kant intenta con la deducción trascendental, sino comprender el sentido de objetos no dados en la experiencia, mientras otros, los objetos pensados por los conceptos empíricos, están dados en la experiencia. El primer problema es cómo establecer esa diferencia. No hay duda de que este planteamiento está hecho partiendo de los logros de la Dissertatio de $1770^{2}$. La Dissertatio distingue los objetos uti apparent, (Sectio II $\$ 4$ ) los objetos fenoménicos, y los objetos sicuti sunt, los objetos tal como son; puesto que no aparecen como son, lo que son sólo puede ser objeto del pensamiento, por eso son objetos del pensamiento, nunca objeto de la sensibilidad. Sin embargo, la realidad objetiva de estos conceptos radica, según se desprende de lo anteriormente dicho, no en el entendimiento sino en la realidad, más aún, en la verdadera realidad. La pregunta por el origen y justificación de estos conceptos no parece estar resuelta en la Dissertatio, que se orienta en una dirección que casi se podría decir innatista. Si el entendimiento no abstrae esos conceptos de la sensibilidad, ¿cómo los posee? ¿y cómo, si no son abstraídos de la sensibilidad, que es el único contacto que tenemos con la realidad, coinciden esos conceptos de la Dissertatio con lo que realmente son las cosas? La solución de Kant es conocida: recurrir a un ejemplar, a su vez ejemplar de las cosas: esos conceptos, dirá Kant, «exeunt in exemplar aliquod... quod est perfectio noumenon", $y$ en este caso es el "Ens summun" (Sectio II, $\$ 10)^{3}$.

Parece evidente que en la introducción a la deducción trascendental se está operando con el mismo esquema. Veremos efectivamente que Kant opera con

2 De la Dissertatio hay una edición bilingüe de Ramón Ceñal, Immanuel Kant, la «Dissertation de 1770. Edición bilingüe, 1961, 21996, CSIC, Madrid.

${ }^{3}$ Ceñal traduce 'exeunt' como use constituyen en un cierto ejemplarw; yo creo que es una traducción incorrecta, que les despoja del dinamismo que está en el acusativo. Yo lo traduciría: use proyectan [salen] hacia un ejemplar* que en definitiva es el Ser supremo. 
un planteamiento crítico y con otro precrítico. Ya el hecho de hablar de objetos dados y objetos no dados en la experiencia hace pensar en dos tipos distintos de objetos, aparentemente como se hacía en la Dissertatio, es decir, objetos uti apparent y objetos sicuti sunt. Hay que advertir de antemano que la Critica kantiana superará esta distinción y el planteamiento precrítico. La deducción trascendental nos ha de mostrar si esos conceptos puros tienen validez y, si piensan un objeto, qué tipo de objetos es el que piensan y en qué relación están esos objetos con los objetos de los conceptos empíricos. Téngase en cuenta que mientras hablamos así estamos aún en el planteamiento precrítico.

Para no perdernos en las expresiones es necesario no olvidar que el concepto es pensamiento de un objeto. Esto, de momento, sólo se puede ejemplificar en la experiencia; la cara A de este objeto implica todo el objeto. Así cuando veo la cara A, toda la experiencia anterior queda implicada en esa experiencia concreta, teniendo de ese modo el objeto total. El concepto es pues el pensamiento o la representación del objeto total. En la experiencia reducida a puro dato, es decir, a experiencia efectiva, no se da ese objeto total. Kant siempre tiene presente un esquema sustancialista del espíritu. La sensibilidad es pura pasividad, de ahí la necesidad del entendimiento para pensar el objeto. La experiencia es sensible, material, concreta; según Kant sólo con una espontaneidad intelectual podremos sobrepasar esa materialidad concreta de la experiencia. Un análisis fenomenológico nos muestra la relación implicativa entre el presente y el pasado, una relación puramente pasiva que el dato presente muestra con los datos pasados. Esa implicación desde el punto de vista husserliano se basa en que el dato presente es una posibilidad realizada en la experiencia, una posibilidad realizada por el propio cuerpo y que connota -es decir, que implica - otras posibilidades del propio cuerpo. Es el propio cuerpo el que mantiene esa unidad; y es el propio cuerpo el sostén de la intencionalidad implicativa. Ahora bien, hay que tener en cuenta que este cuerpo no es el cuerpo objetivo, no es el Körper, sino el Leib, como Husserl lo llamó para distinguirlo del cuerpo, y que podríamos traducir por intracuerpo, soma o carne. Desde una perspectiva fenomenológica los conceptos no son espontaneidades del intelecto sino meras asociaciones implicativas que no se realizan en un nivel fisiológico sino en el ámbito de la conciencia, es decir, en el tiempo de la conciencia, cuya base sería un "yo puedo".

Pero se trata de un párrafo de introducción, lo que quiere decir que se desenvuelve en una terminología y una concepción precrítica o dogmática. En esta formulación dogmática se comprende mucho mejor el sentido de la deducción. 
Supongamos, en efecto, la validez de la terminología del $₫ 13$, lo que el lector dogmático da por supuesto. El entendimiento, en el lenguaje de las facultades, piensa un objeto para una experiencia concreta, piensa un objeto para un dato concreto de experiencia. ¿Qué diferencia hay entonces entre un concepto empirico y un concepto puro? El concepto empírico es un complemento que la facultad cognoscitiva aporta al dato simple de la experiencia pero basado en la misma experiencia, es decir, el concepto empírico lo único que hace es aportar asociativamente a este puro dato las experiencias pasadas que este dato implica; lo único que hace es añadir las experiencias pasadas a la experiencia concreta. El concepto empírico sería experiencia concreta actual (o representada) más las experiencias pasadas asociadas a esta experiencia. En el concepto puro, por el contrario, no puede haber otras experiencias añadidas, ya que es un concepto que se usa independientemente de la experiencia. Con eso queda explicado un primer sentido de la deducción trascendental. Para ello Kant ha utilizado un esquema tradicional, la referencia a dos tipos de objetos, unos dados en la experiencia y otros no dados en la experiencia.

\subsection{Necesidad de la deducción trascendental}

Kant pasa ahora a explicar la necesidad de la deducción trascendental. Empieza con la deducción trascendental del espacio y del tiempo; en ella ha demostrado el valor objetivo de estos conceptos. Tal vez convenga repetir brevemente en qué consiste ese valor objetivo, pues en cierto sentido va a servir de modelo para lo que pueda ser el valor objetivo de los conceptos puros y, por tanto, el resultado de la deducción trascendental de los conceptos. Además, quizás convenga clarificar un punto concreto sobre la concepción del tiempo que después nos volverá a salir. En primer lugar, el sentido objetivo del espacio y del tiempo. Este consiste exclusivamente en que tanto el espacio como el tiempo son condiciones de los objetos de la experiencia sensible, pero esas condiciones no pertenecen a los mismos objetos. El espacio y el tiempo no es algo objetivo-empirico, o si se quiere objetual, no es algo objetivo-objetual, sino objetivo-subjetivo. Si en todo objeto tenemos, como nos dice Kant, dos partes, una, la aportada por la experiencia, y otra, la aportada por el sujeto, el espacio y el tiempo pertenecen al sujeto; ahora bien, todo dato de experiencia viene dado en el espacio y en el tiempo; luego estos son la condición de posibilidad de los mismos datos sensibles o de experiencia; son condiciones objetivo-subjetivas de todo lo objetivo-objetual. ¿Por qué deduce Kant que espacio y tiempo son la forma de la sensibilidad, el espacio forma de la sensibilidad externa y el tiempo forma de la sensibilidad interna? 
Kant utiliza el modelo de la percepción interna de nuestros estados, como, por ejemplo, la alegría, el dolor, el bienestar, etc. En este tipo de percepción tengo conciencia de mi estado, el cual puede estar motivado por algún suceso; pero distingo perfectamente el suceso en sí mismo y el estado que provoca en mí.Tengo una doble conciencia; por una parte la del motivo, y por otra la de mí mismo como motivado o en el estado motivado. Kant, de un modo fenomenológicamente discutible, transfiere este modelo a toda representación cognoscitiva. Todo contenido de conciencia aparece como afección de mi estado; si veo una pared blanca, podriamos decir, según Kant, que mi estado se ve afectado por el blanco. La razón para la ampliación de este modelo la ve precisamente Kant en el espacio y el tiempo. $\mathrm{Ni}$ el espacio ni el tiempo pertenecen a figura o lugar alguno, sino al revés, éstos pertenecen siempre a un espacio y tiempo; así, toda representación externa aparece en el espacio y toda representación tanto externa como interna aparece en el tiempo. Dada la pasividad con que Kant pretende definir la sensibilidad, no queda otro remedio que decir que el espacio y tiempo son formas de la sensibilidad.

El espacio no es nada absoluto; la experiencia no nos da el espacio sino sólo datos que se dan en el espacio; tiene que haber por tanto una justificación para la utilización del concepto de espacio; hay que hacer una deducción de este concepto ${ }^{4}$. Esta deducción no puede consistir en una aportación de algo de la experiencia, pues ésta sólo nos dará datos y más datos, aunque sean datos implicados en los primeros. Todos los datos están siempre implicados en el espacio. El espacio es el horizonte de la experiencia, horizonte propio del sujeto, pues hemos visto que no puede provenir de la experiencia. Ahora bien, Kant no tiene posibilidad de indagar el origen o génesis del concepto de espacio partiendo de una definición sustancialista de las capacidades mentales: el espacio es la forma de la sensibilidad externa, algo asi como el color es la forma de una extensión determinada. Si el rojo es la forma de una extensión, toda figura que se dibuje en esa extensión será roja. El espacio viene así definido como algo hecho, Kant no puede demostrar o ir más allá hacia la génesis. El espacio, y lo mismo valdrá para el tiempo, es un apriori formal. A Kant le falta un método adecuado para ver si es posible que incluso un concepto - con las reservas terminológicas requeridas- como el espacio sea un concepto derivado, es decir, que tenga una constitucion, una génesis. La sensibilidad es algo pasivo, que nace con su forma de ser, como le ocurre

${ }^{4}$ En sentido estricto el espacio y el tiempo no son conceptos sino formas de la sensibilidad, pero aquí Kant los emplea como ejemplos de conceptos puros. 
a cualquier ser, que siempre tiene una forma de ser. La forma de ser de la sensibilidad es el espacio y el tiempo. Kant no podría ver que no todo dato proviene de una experiencia externa a nosotros, no podía ver que hay muchos datos que provienen de la relación que nosotros mantenemos con esas cosas externas; más aún, que toda cosa externa nos viene dada precisamente sólo en la medida en que se establezca una relación entre nosotros y la realidad. El espacio también tiene una génesis. Según Kant la geometría es la ciencia del espacio, pero no necesita recurrir a la experiencia; es independiente de la experiencia. Téngase en cuenta que cuanto dice Kant de la relación entre experiencia y espacio no significa otra cosa sino que el espacio es horizonte de la experiencia, es decir, que toda experiencia se da en el espacio; en esa descripción nada se incluye de una definición sustancialista del espacio como forma de la sensibilidad.

La deducción del espacio no es en absoluto necesaria para la geometría, pues ésta encuentra su evidencia en el propio espacio. El espacio y el tiempo, además de ser formas de la sensibilidad, es decir, formas de la intuición, son ellos mismos intuiciones formales, o sea, tienen un contenido verificable intuitivamente, de modo que las relaciones o propiedades del espacio se nos dan de modo intuitivo, directamente. De ahí deduce Kant que no tengo que acudir a la experiencia para comprender los objetos de la geometría, por ello verificar la geometría no requiere una deducción trascendental. Precisamente el hecho de que tenga que decir que los conceptos de espacio y tiempo son intuiciones, es decir, que tienen un contenido dado intuitivamente, le debería haber hecho pensar en una génesis.

Pero el caso de los conceptos puros es otro porque éstos no tienen ninguna intuición en su base, hablan de objetos por predicados del puro pensamiento. Esta frase, aunque difícil de captar en su significado fenomenológico, es fundamental. En parte la hemos explicado anteriormente. Ahora conviene fijarnos en su más estricta literalidad. Sabemos que un concepto se refiere a objetos, "hablas de objetos; es, hemos insistido, el pensamiento de un objeto. Ahora bien, sabemos que el concepto empírico, tal como también lo hemos explicado, habla de objetos mediante datos de la experiencia. Todo lo que de un objeto incluiría un concepto empírico serían datos de experiencia; un concepto empírico nos diría sobre un objeto cosas de la experiencia. Pero ya sabemos que un concepto puro es independiente de la experiencia. De él no se puede decir nunca que habla por predicados de experiencia; su propio objeto no es un objeto de experiencia; entonces ¿qué es? En todo caso ¿qué uso tienen estos conceptos? Pues bien, nos sigue diciendo Kant, estos conceptos hacen problemático el uso mismo del concepto de espacio. 
Sabemos ya que los conceptos puros son problemáticos en cuanto a su validez objetiva, pues sus objetos no son objetos de experiencia. ¿Es que los objetos a que se refieren estos conceptos puros no tienen los límites que nos imponen la experiencia y la intuición formal? Aparentemente parece que no, pues parece que no piensan objetos del espacio o en el espacio. Mas, por otro lado, todo objeto que el hombre conoce se da en el espacio y en el tiempo. La admisión de estos conceptos puros hace problemático el mismo concepto de espacio, o por lo menos, la relación de las facultades humanas, la relación de la sensibilidad y el resto de las facultades. En todo caso la existencia de los conceptos puros obliga a volver a pensar la deducción del espacio, pues convierten a este concepto en problemático, al ser problemático el objeto que estos conceptos piensan. Los conceptos puros, o si se quiere, la deducción de los conceptos puros hace que la anterior deducción del espacio, la de la estética, esté supeditada a ésta. Mientras en la sensibilidad se comprende que todo objeto está supeditado a la forma, el horizonte que lo constituye, no se ve así con respecto a los conceptos puros. El espacio y el tiempo son condiciones objetivas-subjetivas de la objetividad y en eso exclusivamente radica su objetividad; los conceptos puros, por el contrario, no pertenecen a la sensibilidad, pues nada tienen que ver con la experiencia, ya que piensan objetos no dados en la experiencia. Los conceptos puros son funciones del pensamiento, y sin ellos puede haber fenómenos de la sensibilidad, es decir, se nos pueden aparecer objetos sin que necesariamente tengan que estar referidos a los conceptos puros, como si los conceptos puros no fueran necesarios para la experiencia.

\subsection{Ejemplificación en el concepto de causa}

$\mathrm{El}$ concepto científico de causa significa que, puesta una cosa ' $\mathrm{B}$ ', hay que poner otra distinta ' $A$ ': puesto el efecto hay que poner la causa. Fenomenológicamente tenemos todo lo perteneciente a ' $B$ ', es decir, aquellas experiencias implicadas como posibilidades corporales en torno a este objeto mismo. Pero partiendo de ahí, ¿por qué ha de quedar implicado otro objeto distinto, ya que, como nos dice el propio Kant, la causalidad es una función de alteridad? Para la aparición de ' $B$ ' en el espacio no se requiere 'A'; sin embargo, para Kant no depende sólo de mi experiencia arbitraria el poner o no poner ' $A$ ', sino que puesto ' $B$ ', pongo ' $A$ '; ' $B$ ' implica necesariamente ' $A$ '; pero esa implicación no es necesaria en la experiencia porque ésta no trabaja con necesidad, pero para la objetividad cientifica es imprescindible y necesario. La ciencia, según Kant, no dice que según mi experiencia es probable que ocurra así, sino que lo dice categóricamente. 
En primer lugar, conviene hacer una reflexión en torno a la epistemología kantiana, sobre su concepto de ciencia. En la vida ordinaria, para los asertos, para las predicciones de acontecimientos, nos basamos en la experiencia. Esta experiencia es lo suficientemente estable para que, en el mencionado caso, puesto ' $B$ ' tengamos que poner ' $A$ '. En la experiencia ordinaria tenemos suficientes puntos de anclaje para asegurarnos una predicción que nos oriente en la acción concreta; mi praxis concreta se basa en estas predicciones. Ahora bien, según Kant, la ciencia no se contentaría con este sentido pragmático, sino que pretendería mucho más. Según Kant la ciencia pretendería hacer afirmaciones válidas sobre la realidad causal, es decir, sobre la necesidad que une a determinados fenómenos; ya no se trataría de que dada mi experiencia hay que esperar que sea así, sino que sencillamente es así. Puesto ' $B$ ', se da 'A'; esta implicación de experiencia se da para la ciencia como una implicación de realidad. No nos vamos a meter en la epistemología actual. Sólo me interesa hacer notar un problema concreto, que en el ámbito de lo más inmediato, como pueden ser las afirmaciones más primarias de la fisica o de la astronomía, las afirmaciones científicas se presentan con el sentido de llegar verdaderamente a la realidad, no sólo de hacer proposiciones referidas a una relación pragmática. La ciencia no sólo amplía horizontes de experiencia para darme posibilidad de nuevas formas de acción, sino que, en aquellos numerosos casos en los que los científicos están de acuerdo en la suficiencia de medios de prueba, está segura de la veracidad de sus formulaciones. La ciencia normalmente opera con concepto de necesidad. $Y$ la pregunta de Kant es de donde procede ese postulado de necesidad si no puede provenir de la experiencia.

Es curioso observar que Kant argumenta de modo parecido a Husserl en la critica de la experiencia: la experiencia presenta una marcha concreta, puesto ' $B$ ', ponemos ' $A$ '; puesto ' $B$ ', implicamos una serie de fenómenos, pero en cualquier caso puede introducirse un caos en la serie de fenómenos si atendemos sólo a la sensibilidad. En cualquier caso puede acaecer una Vervirrung (B-123), pero a pesar de ello seguiriamos teniendo objetos de la sensibilidad. Sería interesante exponer detenidamente este punto de la critica husserliana de la experiencia, la posibilidad de un caos de sensaciones, sin que por ello se pierdan los objetos de las sensibilidad. Esto indica que la sensibilidad no necesita del entendimiento para darme objetos.

\subsection{Necesidad de la deducción para la epistemología}

Tratemos ya de resumir los logros hasta ahora alcanzados. Todo parte de una distinción que Kant ha introducido entre conceptos empíricos y concep- 
tos puros. Conceptos empíricos son datos, conjunto de datos de experiencia implicados en un dato presente o representificado. Los conceptos empíricos son una síntesis implicativa de datos. No así los conceptos puros, ya que aportan un contenido a un dato. Tomemos como ejemplo el concepto de sustancia; si me fijo en este dato, el color concreto que veo delante de mí, este color como dato implica otros muchos datos sensibles, por ejemplo, implica el mismo dato hace un rato, el mismo dato desde otra perspectiva, una resistencia manual, es decir, presenta una correspondencia con los datos de otro órgano sensorial (todo lo que se nos da mediante un sentido implica de una forma u otra la posibilidad de traducción a otro sistema sensorial). Toda esta implicación es algo que pertenece a ese dato. Ahora bien, sobre estos datos yo añado algo muy distinto, el "OBJETO", este objeto único y, yendo más allá, el hecho de que este objeto dependa además de otro objeto al que llamo "CAUSA". Ni el objeto único al que pertenece ese dato, o esa serie de datos implicados, ni la relación causal de uno respecto al otro son algo que nos dé la experiencia o la sensibilidad; sin embargo, yo no puedo menos de percibirlos, de afirmarlos y de juzgarlos. Mi experiencia sensible no me la da, es decir, no tengo posibilidad de traducirla a un sistema sensorial, luego esa aportación proviene de mi espontaneidad. Esto supone, por supuesto, el prejuicio de las facultades: la sensibilidad llega y alcanza exclusivamente hasta lo dado pasivamente, no nos da más, siendo el entendimiento el que ha de aportar el resto, lo espontáneo, lo no sensible. Ahora bien, en cuanto la ciencia se basa en estos conceptos, la deducción de su valor y la legitimación de su uso es imprescindible para la misma ciencia. Por tanto, la epistemología incluye por necesidad una deducción. Lo que está pues en juego es el valor de la ciencia, de una ciencia considerada como lugar de la verdad, de la verdad en sentido tradicional y que, por ello, tiene que justificar sus conceptos, ya que la regularidad fáctica de la sensibilidad no basta. En segundo lugar está en juego el valor de la experiencia en virtud de la cual afirmamos los juicios científicos. Esta experiencia hemos visto que, según Kant, incluye elementos sensibles y elementos no sensibles. Por eso para Kant la experiencia desborda la percepción; la percepción sólo incluye lo sensible, mientras la experiencia incluye también los conceptos puros no sensibles, provenientes de la espontaneidad del entendimiento.

En la deducción trascendental se trata, en definitiva, de la constitución del objeto, ya que toda percepción actúa con objetos, no con meros datos, pues incluso los datos empíricos básicos del concepto empírico van más allá del mero dato, afirmando un objeto. Evidentemente ya con este planteamiento se relativiza el mismo concepto de sensibilidad, pues desde esta perspectiva la sensibilidad ya no es algo independiente como Kant pretendía hacer ver al principio. 


\subsection{Conclusiones de este párrafo}

Consideremos, en primer lugar, el planteamiento básico kantiano. En relación con esto podríamos decir que en Kant, en concreto en este párrafo, se puede ver una reducción muy parecida a la del propio Husserl. La reducción de Husserl es la vuelta al sujeto trascendental, debido al hecho de que todo lo conocido es una constitución del sujeto cognoscente, de que no podemos salirnos del ámbito del sujeto de la vida mental-psíquica en la que aparece el mundo. En esta reducción nos encontramos con unos datos hiléticos como factum contingente e irreducible, a partir de los cuales 'se organiza el mundo', o 'se constituye el mundo'. Kant practica la misma reducción, aunque basado en presupuestos tradicionales; la sensibilidad sólo me da algo pasivamente; la experiencia desborda lo hilético; luego lo que hay de más debe provenir del sujeto; hay que llevar, pues, la experiencia a su origen, hay que descubrir su constitución.

No se trata, en segundo lugar, sólo de hallar la constitución de la experiencia cientifica, pues la experiencia cientifica no tiene en su base operaciones cognoscitivas cualitativamente distintas de la experiencia ordinaria. La teoría del conocimiento de Kant se aplica a todo conocimiento, aunque utilice Kant precisamente la ciencia, por ser en la ciencia donde se pretende conscientemente conseguir la objetividad; pero, también en la experiencia ordinaria trabajamos o actuamos con un principio de objetividad. Si en un movimiento arriesgado dudara de la seguridad que me puede proporcionar mi experiencia seguro que no lo haría. La deducción trascendental afecta al conjunto de la experiencia o al conjunto del conocimiento de experiencia propio del ser humano.

Tercero, el punto de partida de Kant se hace desde el prejuicio de las facultades, en el lenguaje tradicional de las facultades del alma, es decir, desde un modelo antropológico tradicional, dependiente todavía de la tradición metafísica. Precisamente este concepto tradicional es lo que le impidió abordar un correcto análisis del espacio y tiempo, sobre todo del espacio, a partir de la vivencia del cuerpo en su relación con las cosas de la experiencia. Un análisis del concepto de espacio sin prejuicio de facultades le hubiera llevado a analizar el contenido del espacio buscando su génesis y no dándolo por supuesto como forma de una facultad.

Y cuarto, junto con este prejuicio del lenguaje de las facultades se da lo que podemos llamar un prejuicio sobre el sentido de uobjetividad", tanto de la ciencia como de la experiencia. Según Kant la realidad objetiva va más allá de los 
datos de la sensibilidad, es decir, de los datos de la percepción, de lo hilético. La percepción no nos da objetos, sino datos pasivamente asociados, sin ningún tipo de profundidad objetiva; esta profundidad objetiva no la da la experiencia; luego la objetividad ha de venir de mí, no puede venir de mi contacto con la realidad. Esta objetividad se manifiesta en los conceptos puros. Son estos conceptos puros los que hay que analizar y seguir hasta su origen en el sujeto, pues la percepción no nos los puede dar.

En el desarrollo de la deducción veremos hasta qué punto Kant supera esos prejuicios y planteamientos para llegar a modificar el sentido mismo de objetividad.

\section{3. § 14: paso a la deducción trascendental de las categorías}

\subsection{Origen precrítico de la distinción entre conceptos puros y conceptos empíricos}

El planteamiento tradicional con el que Kant empieza la deducción se ve de modo aún más claro en el comienzo de este parágrafo: hay dos formas de relacionarse las representaciones y sus objetos, o «es el objeto el que hace posible la representación", "o es ésta la que hace posible el objeto". La relación en el primer caso es empírica; como el segundo está prohibido a la finitud humana, introduce Kant otra modalidad, que será la médula espinal de toda la teoría crítica de la constitución de la objetividad según Kant. Antes de ver esta tercera posibilidad nos interesa detenernos en el planteamiento como tal.

Kant distingue entre representación en general y su objeto; el objeto parece quedar fuera de la representación, la representación dentro de mí; por ejemplo, el concepto del libro es mi representación del libro real; esta representación se relaciona a su objeto, porque pensamos que éste la ha producido y de ahí que entre mi representación y el libro haya una coincidencia, se relacionan por la producción: el objeto hace posible la representación. ¿QQué es lo que esta descripción capta del conocimiento sensible, puesto que a él se refiere? Hemos descrito el sentido fenomenológico primario del concepto empírico. Veamos ahora qué aspecto del fenómeno es captado con esta terminología. Un concepto empírico es el resultado de una implicación entre un dato presente y una serie de datos pasados o futuros que han sido o serán percibidos; la representación conceptual desborda el dato presente. ¿Puedo distinguir entre el dato presente 
aquí y mi representación del dato? Fenomenológicamente no hay ninguna distinción. La distinción fenomenológica se da entre el dato presente y lo implicado no presente. En cuanto el concepto abarca más que el dato presente, desborda este dato; mas ese desbordamiento no es sino el hecho de que mi vida concreta desborda mi presente, como el instrumento desborda su aplicación concreta, en la medida en que sirve para todas las veces que se me presente la misma oportunidad; o como la palabra desborda su uso actual. Mi vida concreta desborda mi presente puntual, en cuanto el presente se constituye por el pasado, de ahí que el concepto empírico no pueda ser separado del objeto, porque es pensamiento de un objeto; sólo puede separarse la presencia del dato presente y los demás datos posibles, la presencia del concepto dado con el dato presente y la de los componentes del concepto son diversas, porque, el objeto tiene diversas presencias, una parte es presente, mientras las demás no, sino sólo implicadas, o porque fueron presentes en su momento, o porque pueden hacerse presentes en el futuro.

Ahora bien, entre el objeto real caracterizado por propiedades reales, hay algo, el dato presente, que depende de mí, implicando por eso todo el sistema de presencia humana, porque se escorza, tiene perspectivas, implicaciones de experiencias anteriores, lingüísticas, etc. y nada de esto muestra características de lo que entendemos por objeto real, pues la presencia de éste es presencia en el mundo, y no es, por lo tanto, presencia humana. Un problema muy interesante sería distinguir ambas presencias'. Tenemos pues un concepto, este dato presente más todas las implicaciones de datos que están en él, el cual se refiere a ese objeto real que tiene propiedades no propias de la representación, pues mientras el libro real se puede quemar, nunca se podría quemar la representación que de él tengo en perspectiva o en el recuerdo, ni las implicaciones de los datos; su presencia es de otro nivel que la presencia mundana; precisamente este fenómeno es lo que capta la descripción kantiana, la diferencia entre el nóema (la representación) y el objeto real; el concepto es el nóema que se refiere a un objeto real. El problema de la objetividad consistirá en saber cómo se puede pasar del nóema-subjetivo, pues en todo caso depende del sujeto, al objeto real, con las características propias de la

${ }^{5}$ Fink acuñó los términos Anschein, para la presencia o fenómeno en el mundo, y Vorschein, para la presencia o fenómeno de algo ante un sujeto. Cf. Fink, Sein, Wabrheit, Welt, M. Nijhof, La Haya, 1958. Hay traducción de este texto en la Revista Episteme. Anuario de Filosofía. I, Caracas, 1957 pp. 177-314. 
realidad, a saber, resistencia, causalidad, influjo y composición de fuerzas, etc., y que por lo tanto se puede destruir por la influencia de otros cuerpos.

Pero Kant va más allá; no ve problema alguno en los conceptos empíricos, cuando en realidad el mismo contenido de objetividad presentarán los conceptos empíricos que los otros. El objeto real como algo distinto del nóema, del concepto, es tan problemático como los conceptos puros, y posteriormente lo veremos con la deducción, que, en efecto, si los conceptos empíricos presentan un grado de objetividad, (cf. 159 (A-109)) realidad, sustancia, etc. es porque en el nóema empírico se constituye también una objetividad. La diferencia radica en que los contenidos noemáticos empíricos no presentan problema al depender de datos hiléticos irreductibles, mientras que los conceptos puros no son datos hiléticos, sino modos de objetividad de lo real. Con esto vemos que lo afectado por la deducción es la totalidad de la experiencia.

El punto de partida sin embargo es el tradicional, que sitúa el concepto empírico exclusivamente en el nivel de la percepción y el concepto puro en el nivel del intelecto, llamándo sin embargo concepto a ambos. Ahora bien, tal como los hemos descrito, los conceptos empíricos, como datos que implican otros datos, no serían, desde el punto de vista de Kant y hablando propiamente, conceptos, es decir, el pensamientos de un objeto, porque dependen sin más de la experiencia, en ese sentido son sólo datos implicados. Ahora bien, en cuanto esos "conceptos" o datos implicados se refieren a un objeto, lo que es evidente por el uso que de ellos hacemos, un objeto además que ha de ser real, entonces el nóema como concepto empírico ha de ser ayudado por otro tipo de conceptos, que son conceptos en sentido propio, como condiciones mediante las cuales es posible conocer algo como un objeto, es decir, pensar un objeto. El concepto empírico por sí mismo no piensa un objeto; de ahí que no tengan ambos 'conceptos' el mismo sentido y que no se pueda decir que el concepto empírico se relaciona a su objeto porque éste lo hace posible; propiamente hablando el concepto empírico sólo se relaciona a un dato presente en una relación de implicación. La distinción de conceptos empíricos y puros, ambos igualmente como pensamientos de objetos para una experiencia, tiene un origen dogmático en el Kant precrítico que el Kant de La crítica tendrá que superar. Evidentemente sobre esta caracterización de los conceptos puros se deberá volver repetidas veces, pues ahl radica el núcleo de la crítica, en ser condiciones de posibilidad de pensar un objeto a la experiencia, o, si se quiere, a los - mal llamados- conceptos empíricos. 


\subsection{La relación cognoscitiva como una relación trascendental}

De las tres posibilidades antes citadas, la primera se nos ha mostrado problemática, pues el sentido de objetividad de los conceptos empíricos sólo se comprende dentro de una perspectiva tradicional dogmática, y en un esquema tradicional antifenomenológico. La segunda posibilidad debería haber sido analizada por Kant, pues presenta la humana posibilidad del trabajo, pero es tomada por Kant en el sentido de la tradición cristiana de la creación, es por tanto rechazada como imposible para el ser humano. Lo nuevo y fundamental es, pues, la tercera, que es la que más nos interesa. En resumen, dice, sólo por la representación podemos conocer un objeto (B-125). Tenemos que analizar a fondo esta posibilidad porque en ella se presenta el carácter fenomenológico de la solución kantiana de la constitución de la objetividad, aunque la exposición ciertamente se haga en un ambiente o contexto más bien deductivo que fenomenológico. Hemos visto ya la primera posibilidad, que, a pesar de captar el fenómeno o un fenómeno, no describe fielmente el carácter objetivo de los conceptos empíricos; en todo caso esa posibilidad no es la propia de la objetividad, porque en ésta se da un salto (Sprung) del dato presente, es decir, de la presencia humana, al ser real objetivo, a una presencia cósmica, que desborda la mera presencia humana.

La segunda posibilidad es rechazada por antifenomenológica, nunca se da el caso de que la presencia humana produzca el objeto real. Esto que es una modificación del hecho real del trabajo, no es propiamente hablando posibilidad fenomenológica; de ahí que no quede sino la tercera, que yo constituya la objetividad para el dato presente y sus implicaciones, para el llamado concepto empirico en su sentido fenomenológico. No sería fenomenológico decir que el dato presente por ser presente es ya objetivo, porque la presencia cósmica no es igual a la presencia humana, y yo sólo tengo presencia como cosas presentes a mí. Lo a mí presente implica en su mismo modo de ser una serie de datos y complejidades que lo constituyen como tal, pero que no implican el ser presente cósmico. ¿Cómo puedo pasar de mi ser presente, de mi concepto empírico, a la presencia cósmica, a la existencia real objetiva en el mundo? Puesto que todo lo presente a mí es relativo a mí, es decir, es propio de mi vida intencional implicando todo lo que esta vida intencional le presta, nunca puedo salir de él.

Fenomenológicamente no puedo salir fuera de mi vida intencional. Tampoco puedo decir que lo presente a mí es sin más presente en el mundo. La presencia cósmica, la objetividad del dato presente, repreșenta un Überschuss [desbordamiento] respecto al dato presente, pero este Überschuss no puede radi- 
car fuera, sino en el mismo dato presente, de ahí que la objetividad no sea 'producida' por la presencia cósmica sino sólo por mí. La objetividad proviene del mismo sujeto, es una organización de lo que me es presente por la cual yo sobrepaso el nóema relativo a mí, los conceptos empíricos, 'pensándoles un objeto'; los datos empíricos presentes por sí mismos no son objetivos, sólo son objetivos en sentido intencional; son algo dependiente de mí; la objetividad propiamente dicha es el pensamiento de un objeto para esos datos. Un objeto será el conjunto del dato presente -implicando todo lo que se llama concepto empírico-, y de un concepto puro que 'piensa' un objeto para ese dato presente, que como tal dato no nos puede dar más que el dato. Kant se corta ya desde este $\$ 14$ todo recurso metafísico, reduciéndose exclusivamente al análisis de ese hecho, cómo pensamos un objeto para los datos empíricos y por qué pensamientos míos han de ser o pueden ser precisamente el valor de objetividad que los datos llevan consigo y que consiste en hacer que ese dato no sea sólo una presencia mía sino una presencia cósmica, es decir, una realidad cósmica.

Una vez anunciada esta tercera posibilidad sigue una nueva indicación sobre las dos condiciones de la experiencia, la intuición y los conceptos, aquella que nos da el dato presente, y éstos que piensan objetos para esos datos. Kant ha demostrado ya que los datos presentes se dan según las formas de la intuición, el espacio y el tiempo. Ya se ha insinuado el sentido fenomenológico de esto no elucidado por Kant, sino expuesto en los términos de un lenguaje de facultades. El espacio y tiempo son las condiciones de posibilidad de los datos mismos. Ahora se quiere ver si, además del espacio y tiempo, se requieren conceptos puros, es decir, puros pensamientos de objetos para que se den objetos de experiencia; fenomenológicamente parece que se requieren, como se ve en los análisis anteriores, que son admirablemente resumidos ahora por Kant. Toda experiencia además de intuición tiene concepto que le piensa un objeto en el sentido ya explicado; de ahí que a priori tengamos conceptos de objetos; estos conceptos son las categorías. Su valor objetivo consiste precisamente en ser condiciones de posibilidad de pensar objetos para las intuiciones, es decir, sólo por ellos es posible la experiencia, por tanto, incluso los conceptos empíricos, pues sólo por aquellos se puede pensar un objeto para los conceptos empíricos.

\subsection{La deducción objetiva de la primera edición}

En el $\$ 13$ ha mostrado Kant el sentido de la deducción desde una perspectiva jurídica: ver la validez objetiva de los conceptos, su derecho. Las intuiciones 
por sí mismas sólo se atienen a las condiciones espacio-temporales; por sí mismas podrían suceder en una 'Verwirrung', una confusión, en sí mismas no necesitan de conceptos puros; ahora bien, la experiencia nos muestra una regularidad, luego de facto las intuiciones o los datos presentes tienen un desbordamiento sobre ellos mismos, es decir, están sometidos a los pensamientos de objetos que son las categorías; éstas posibilitan la experiencia objetiva; con esto ya se ha dicho todo respecto al planteamiento primero de la deducción trascendental; a la pregunta por el derecho de los conceptos puros responde Kant que son objetivos porque sólo por ellos pensamos objetos para las intuiciones, para los datos presentes. Con esto se descubre el principio según el cual ha de seguir toda la "Nachforschung", investigación posterior: son condiciones a priori de la experiencia; es decir, toda experiencia de objetos, que es la experiencia ordinaria, se da sólo porque somos capaces de pensar objetos a los datos presentes, no sólo pues de asociarlos a las implicaciones de otras experiencias, sino también de pensarles un desbordamiento que ya no es dato presente, ni puede serlo.

Con esto parece terminada la deducción objetiva de las categorías tal como se nos indica en la introducción a la primera edición, donde dice expresamente (A, XVII), hablando de las páginas 92 y 93 , que al que no parezca convencer la deducción subjetiva presentada en las otras dos secciones, que acepte la primera, que es la fundamental.

Ahora bien, ętermina Kant con esto la deducción trascendental? Hay que contestar afirmativamente si consideramos el sentido jurídico definido en el \$13; pero no es así según los cometidos de una crítica total de la razón, porque se trata ya no sólo de definir el valor objetivo de las categorías que en el análisis -que, una vez aceptado el terreno de juego de la terminología 'categoría', puede ser considerado fenomenológico- se muestran como objetivas por ser pensamientos de objetos para la intuición, para los datos de la experiencia, sino que se trata además de analizar el valor de la objetividad. Yo pienso la experiencia como objetiva. Eso es un hecho. Pero ¿qué significa en realidad pensar un objeto? ¿qué valor tienen las categorías? Porque decir que tienen valor objetivo aún no es decir mucho, porque se trata de ver qué significa el valor objetivo. Se trata de ver ahora porqué a las intuiciones se les piensan siempre unos determinados objetos y qué valor tienen estos objetos pensados. ¿Pensamos en efecto la propia realidad con algún derecho o estamos encerrados en nuestro propio pensamiento sin poder testificar en cada presencia humana una presencia cósmica? El desarrollo de la deducción trascendental consiste en ver de forma concreta cómo se 
da ese valor objetivo de las categorías y, por tanto, en qué consiste ese valor objetivo, cómo pensamos un objeto a los datos presentes, cómo lo tenemos que pensar y, en consecuencia, cómo los datos presentes ya han de ser pensados objetivamente. En realidad lo que Kant llama deducción objetiva nos dice muy poco, tan poco que no es sino una introducción al verdadero núcleo de la deducción.

En la deducción trascendental se trata pues de varios puntos: 1) El valor objetivo de las categorías puras. 2) El modo cómo pensamos objetos a los fenómenos. 3) El sentido mismo del objeto que es pensado, es decir, no ya el valer como objeto, sino el valor del objeto pensado. 4) El uso legítimo del concepto puro, es decir, los límites de la razón. 5) Como resumen, la constitución de la objetividad en su doble vertiente de constitución subjetiva y constitución racional. El \$14 sólo responde al punto primero, que es lo que Kant llama deducción objetiva. Los otros puntos son estudiados y contestados en las otras dos secciones de la deducción trascendental; el punto 2) se responde fundamentalmente en la deducción subjetiva, a la cual da paso en el último párrafo de este $\$ 14$ de la primera edición, al hablarnos de las tres facultades que son las condiciones de la experiencia: sentido, imaginación y apercepción, en las cuales se fundan la sinopsis de lo múltiple a priori, la síntesis de esta multiplicidad por la imaginación y la unidad de esta síntesis por la apercepción, las cuales pueden ser usadas empírica y trascendentalmente; de todo ello tratará la deducción subjetiva, cuya meta es ver cómo conocemos o pensamos un objeto a las intuiciones. 